DOI: $10.1002 /(($ please add manuscript number $))$

Article type: Communication

\title{
Low-Cost and Sustainable Organic Thermoelectrics Based on Low- Dimensional Molecular Metals
}

By Florian Huewe*, Alexander Steeger*, Kalina Kostova, Laurence Burroughs, Irene Bauer, Peter Strohriegl, Vladimir Dimitrov, Simon Woodward and Jens Pflaum*

F. Huewe $e^{[\dagger]}$, A. Steeger ${ }^{[\dagger]}$, Prof. Dr. J. Pflaum

Experimental Physics VI

Julius-Maximilian University of Würzburg

and Bavarian Center for Applied Energy Research (ZAE Bayern e.V.)

97074 Würzburg, Germany

E-mail: fhuewe@physik.uni-wuerzburg.de, asteeger@physik.uni-wuerzburg.de, jpflaum@physik.uni-wuerzburg.de

Assoc. Prof. K. Kostova, PhD, Prof. V. Dimitrov, DSc.

Institute of Organic Chemistry with Centre of Phytochemistry

Bulgarian Academy of Sciences

Sofia 1113, Bulgaria

Dr. L. Burroughs, Prof. Dr. S. Woodward

GSK Carbon Neutral Laboratory for Sustainable Chemistry

University of Nottingham, Jubilee Campus

Nottingham NG7 2GG, United Kingdom

I. Bauer

Experimental Physics II

University of Bayreuth

95440 Bayreuth, Germany

Prof. Dr. P. Strohriegl

Macromolecular Chemistry I

and BIMF

University of Bayreuth

95440 Bayreuth, Germany

${ }^{[\dagger]}$ These authors contributed equally to this work.

Keywords: organic thermoelectric materials, molecular metals, radical ion salts, thermal conductivity, Seebeck coefficient

More than $70 \%$ of the primary energy consumed world-wide is wasted, mostly as heat below $100{ }^{\circ} \mathrm{C}^{[1]}$. Thermoelectric generators may convert a substantial amount of this energy into electrical power but high production costs and scarcity of efficient thermoelectric materials operating in this temperature regime have limited large-scale applications so far. Recently, conducting polymers have 
been proposed as potential candidates to meet these challenges showing appreciable low-temperature thermoelectric performance, but unfortunately suffering from low electrical conductivity due to inherent disorder ${ }^{[2-5]}$. Herein, crystalline low-dimensional molecular metals are demonstrated as an alternative class of thermoelectric materials combining the advantages of low weight, chemical variety, sustainability and high charge carrier mobility with reduced electronic dimensionality. For the first time determining all relevant thermoelectric quantities on individual organic crystals of both, p-type $\mathrm{TTT}_{2} \mathrm{I}_{3}$ and n-type $\mathrm{DCNQI}_{2} \mathrm{Cu}$ conductors, high power factors and promising figures of merit surpassing values of $z T \geq 0.15$ below $40 \mathrm{~K}$ are disclosed in this study. The cost-defining power output per active area of a prototypical, all-organic TEG takes unprecedented values of $\sim \mathrm{mW} / \mathrm{cm}^{2}$ at RT. Violation of the Wiedemann-Franz law and phonon drag effects emerge from the materials' lowdimensionality and are expected to deliver further thermoelectric enhancement feasible in near future.

The dimensionless figure of merit $z T$ measures a material's thermoelectric performance

$$
z T=\frac{S^{2}}{\rho \kappa} T=\frac{\sigma S^{2}}{\kappa} T=\frac{P F}{\kappa} T
$$

where electrical conductivity $\sigma$ (resistivity $\rho$ ), Seebeck coefficient/thermopower $S$, and thermal conductivity $\kappa$ provide the power factor $P F=\sigma S^{2}$. Materials with high electrical conductivity and thermopower but low thermal conductivity are desirable to maximize $z T$ and hence, the thermoelectric conversion efficiency. Approximately, $S$ is inversely proportional to $\sigma$ which in turn is related to the electronic thermal conductivity $\kappa_{\mathrm{el}}=\sigma L T$ via the Wiedemann-Franz (WF) law. Advances in $z T$ have been achieved by tuning the doping of semiconductors to maximize the $P F^{[6]}$ together with reducing the phononic contribution to thermal conduction by means of nanostructured superlattice architectures ${ }^{[7,8]}$. To overcome the inherent $z T$ limitations of ordinary materials, a dimensionality reduction of the electronic system has been proposed ${ }^{[9]}$. This can lead to a violation of the WF law ${ }^{[10,11]}$ and to phonon drag contributions to the thermopower ${ }^{[12]}$. Complex crystal structures, e.g. Zintl compounds ${ }^{[13]}$ and skutterudites ${ }^{[14]}$, have been evaluated as good thermoelectric 
candidates due to reduced lattice thermal conductivity and electronic band structure tunability. These optimization strategies, together with the ability for low-cost production and low-temperature processibility, have recently raised interest in organic polymers ${ }^{[15]}$, such as PEDOT:Tos, reaching $z T=0.25$ at room temperature $(\mathrm{RT})^{[4]}$ and thus advancing the value of $z T=1.2$ obtained for $\mathrm{Bi}_{2} \mathrm{Te}_{3}$ (the best RT thermoelectric material to date ${ }^{[7]}$ ). Herein, quasi-1D organic conductors based on small molecules constitute a new, sustainable approach towards organic thermoelectrics that provide many of the desired electrical and thermal properties described above. Additionally, they are lightweight and thermodynamically stable allowing for portable device manufacturing and long-term usage. In comparison to organic polymers the availability of electrically high-performing $\mathrm{p}$ - and ntype organic conductors facilitates the construction of all-organic thermoelectric devices.

As a starting point we chose two of the best p- and n-type conducting radical ion salts, $\mathrm{TTT}_{2} \mathrm{I}_{3}(\mathrm{TTT}$ $=$ Tetrathiotetracene $)$ and $(\mathrm{DMe}-\mathrm{DCNQI})_{2} \mathrm{Cu}(\mathrm{DMe}-\mathrm{DCNQI}=$ Dimethyl-Dicyanoquinonediimine $)$. They form macroscopic needle-like single crystals as depicted in Figure $\mathbf{1}$ together with their respective crystal structures. In $\mathrm{TTT}_{2} \mathrm{I}_{3}$ the molecules stack face-to-face along the needle axis providing large orbital overlap along the $\vec{b}$-direction. These stacks are separated by triiodide counterions impeding a delocalization of charge carriers in the transverse directions resulting in anisotropic transfer integrals of $\frac{t_{\|}}{t_{\perp}} \approx 56^{[16]}$. By charge-transfer of $1 / 2 e$ from each TTT molecule to the iodine atoms, the one-dimensional valence band formed by the HOMO of TTT becomes partially occupied by holes characterizing the material as a p-type organic metal with quasi-1D band structure ${ }^{[16]}$. The interference of two electron-phonon scattering mechanisms in $\mathrm{TTT}_{2} \mathrm{I}_{3}$ has been theoretically proposed to precipitate high charge carrier mobility states and large $z T$ values ${ }^{[17]}$. Furthermore, our low-cost synthesis route for TTT highlights the possibility of sustainable, largescale production (see supplementary information).

In $\mathrm{DCNQI}_{2} \mathrm{Cu}$, the molecules align in separated stacks along the needle-axis ( $\vec{c}$-direction) with a partial charge transfer of $4 / 3 e$ from the central copper atom to two DCNQI molecules. With a conduction band emerging from the partially filled delocalized LUMO, this organic metal features n- 
type conductivity. In contrast to $\mathrm{TTT}_{2} \mathrm{I}_{3}$, the orbital overlap along the transverse directions is larger and the crystal is characterized as n-type organic metal with $\frac{t_{\| l}}{t_{\perp}} \approx 4$ and anisotropic, but still quasi$3 \mathrm{D}$ band structure ${ }^{[18]}$. In contrast to organic charge-transfer salts constituting of two molecular entities, e.g. TTF-TCNQ, between which charge transfer occurs leading to ambipolar conduction, the materials presented in this study belong to the class of radical ion salts and provide unipolar transport (n-type $\mathrm{DCNQI}_{2} \mathrm{Cu}$; p-type $\mathrm{TTT}_{2} \mathrm{I}_{3}$ ) along the molecular chains in presence of a localized inorganic counterion. Thus, they are expected to show improved thermoelectric performance and define a prototypical platform to investigate the impact of dimensionality on the electrical and thermal material properties.

A fundamental problem in the comprehensive thermoelectric characterization of organic conductors stems from the purity-dependence of material properties as well as their pressure-dependence when measured in different experimental setups and contact arrangements. Here, we present a newly developed apparatus to measure all thermoelectric quantities exclusively on the same specimen under minimum strain between $20 \mathrm{~K}$ and $300 \mathrm{~K}$. The sample holder is illustrated in Figure 2a. With graphite paint the crystals are attached to free-standing copper wires in a 4-probe geometry enabling accurate electrical conductivity measurements. Connecting the copper wires at opposite ends of the sample to two independent heating blocks allows a temperature gradient across the crystal to determine the Seebeck coefficient as described by Chaikin and Kwak ${ }^{[19]}$. Measurement of the thermal conductivity was attained by means of the $3 \omega$-method at self-heating geometry for rodlike samples of high surface-to-volume ratio ${ }^{[20,21]}$. Feeding an AC-current, $I_{0} \sin (\omega t)$, into the sample via the outer contacts results in a temperature and resistance oscillation of doubled frequency for materials with a temperature-dependent resistivity of the form $\rho=\rho\left(T_{0}\right)[1+\alpha \Delta T]$. From this temperature oscillation a voltage drop emanates at the inner contacts containing a component of tripled excitation frequency $3 \omega$ with complex amplitude ${ }^{[20]}$ :

$$
\operatorname{Re}\left[U_{3 \omega}\right]=\frac{\alpha R^{2} I_{0}^{3} l}{48 \kappa A} \frac{1}{1+(\omega \tau / 5)^{2}}
$$




$$
\operatorname{Im}\left[U_{3 \omega}\right]=\frac{\alpha R^{2} I_{0}^{3} l}{48 \kappa A} \frac{\omega \tau / 5}{1+(\omega \tau / 5)^{2}}
$$

Here, $R$ is the resistance of the crystal of cross section $A$ and length $l, \kappa$ the thermal conductivity and $\tau=\mathrm{c}_{\mathrm{mol}} \rho_{\mathrm{dens}} l^{2} /\left(\kappa \mathrm{m}_{\mathrm{mol}}\right)$ the thermal response time allowing for determination of the molar specific heat $c_{\text {mol }}$ if the material's density $\rho_{\text {dens }}$ and molar mass $m_{\text {mol }}$ are known (compare supplementary information).

Figure $2 \mathrm{~b}$ depicts the measured complex $3 \omega$-voltage together with the fits of Equation 2 for a $\mathrm{DCNQI}_{2} \mathrm{Cu}$ crystal at RT: $\kappa$ is determined by the amplitude of the real part extrapolated to zero frequency while $\omega=5 / \tau$ corresponds to the peak position in the imaginary part. The fit yields a thermal conductivity of $\kappa=(1.73 \pm 0.1) \mathrm{W} /(\mathrm{m} \mathrm{K})$ and a molar specific heat of $c_{\mathrm{mol}}=(526 \pm$ 70) $\mathrm{J} /(\mathrm{mol} \mathrm{K})$ for $\mathrm{DCNQI}_{2} \mathrm{Cu}$ agreeing in magnitude with extrapolated literature data ${ }^{[22,23]}$. The measured values were also chosen to simulate the dynamic evolution of the temperature profiles inside the crystal for different excitation frequencies in the applied geometry by means of finiteelement methods (Figure 2c). At low frequencies, i.e. $\omega \ll 5 / \tau$, the temperature oscillates in-phase with the heating current. At frequencies approaching $5 / \tau$, the amplitude of the oscillation decreases and shifts in phase with respect to the periodic stimulus. Additionally, the temperature does not fully relax back at zero-crossing current amplitude. For $\omega \gg 5 / \tau$ the amplitude decreases proportional to $\omega^{-1}$ corresponding to DC-heating of the crystal as its thermal response is too slow. Simulating the complex $3 \omega$-voltage, the amplitude extrapolated to zero frequency perfectly agrees with the measured one, as illustrated in Figure 2b. Thus, the method allows us to determine thermal conductivities with high reproducibility and accuracy. Another benefit is the compensation of errors in the sample dimensions when determining $z T$. For $\mathrm{TTT}_{2} \mathrm{I}_{3}$ we obtained RT values of $\kappa=(3.7 \pm 0.2) \mathrm{W} /(\mathrm{m} \mathrm{K})$ and $c_{\mathrm{mol}}=(1191 \pm 150) \mathrm{J} /(\mathrm{mol} \mathrm{K})$

For both materials the temperature-dependent electrical resistivity $\rho$ together with the thermopower $S$ and the thermal conductivity $\kappa$ is displayed in Figure 3a-c in a temperature regime between $20 \mathrm{~K}$ and $300 \mathrm{~K}$. The $\mathrm{TTT}_{2} \mathrm{I}_{3}$ p-type conductivity of $\sigma_{\mathrm{RT}}=2.1 \cdot 10^{5} \mathrm{~S} / \mathrm{m}$ at $\mathrm{RT}$ is among the highest 
reported for organic solids exceeding values obtained in the organic polymer PEDOT:Tos ${ }^{[5]}$. With $\sigma_{\mathrm{RT}}=10^{5} \mathrm{~S} / \mathrm{m}$ the conductivity of $\mathrm{DCNQI}_{2} \mathrm{Cu}$ also achieves an extraordinary high value for an $\mathrm{n}$ type organic conductor. Furthermore, the quantities are measured across the macroscopic distances $(\sim \mathrm{mm})$ necessary for thermoelectric devices to retain a temperature gradient. The strong temperature dependence of the resistivity $\rho=\rho_{0}+\beta T^{\gamma}$, with $\gamma>1$, is typical for low-dimensional organic metals. The RT Seebeck coefficients $S_{\mathrm{TTT}_{2} \mathrm{I}_{3}}=+42 \mu \mathrm{V} / \mathrm{K}$ and $S_{\mathrm{DCNQI}_{2} \mathrm{Cu}}=-34 \mu \mathrm{V} / \mathrm{K}$ are in agreement with values previously reported ${ }^{[24,25]}$. From the slope of the temperature-dependent Seebeck coefficients a large band width of $W_{\mathrm{TTT}_{2} \mathrm{I}_{3}}=632 \mathrm{meV}$ is estimated for $\mathrm{TTT}_{2} \mathrm{I}_{3}$ enabling high charge carrier mobilities, a key requirement for good thermoelectrics. Another feature of the Seebeck coefficient is its non-zero offset when extrapolating the linear fits to zero temperature. This additional contribution persists at RT. In addition to the diffusive electronic part $S_{\mathrm{d}}$ being linear in temperature, for $\mathrm{DCNQI}_{2} \mathrm{Cu}$ we also considered a phonon drag contribution in the fit (supplementary information):

$$
S=S_{\mathrm{d}}+S_{\mathrm{drag}}=a \cdot T+\frac{D}{T^{c}} \cdot\left(\frac{\theta_{\mathrm{E}}}{T}\right)^{2} \frac{e^{\theta_{\mathrm{E}} / T}}{\left(e^{\theta_{\mathrm{E}} / T}-1\right)^{2}}
$$

From the slope $a$ the band width was determined as $W_{\mathrm{DCNQI}_{2} \mathrm{Cu}}=333 \mathrm{meV}$. The scattering parameter $c=1.2$ is close to the predicted value of 1 for neat phonon-phonon Umklapp scattering. The Einstein temperature of $\theta_{\mathrm{E}}=85 \mathrm{~K}$, characterizing the average energy of heat-carrying phonon modes, agrees with values obtained from a Debye fit of low-temperature specific heat data ${ }^{[26]}$. The tail of the phonon drag extends to anomalously high temperatures accounting for an offset of $-5 \mu \mathrm{V} / \mathrm{K}$ at $\mathrm{RT}$ for $\mathrm{DCNQI}_{2} \mathrm{Cu}$.

The temperature dependence of $\kappa$ for both materials is illustrated in Figure $3 \mathrm{c}$. In $\mathrm{TTT}_{2} \mathrm{I}_{3}$, it qualitatively reflects the electronic thermal conductivity as calculated from the WF law $\kappa_{\mathrm{el}}=\sigma L_{0} T$, with $L_{0}=\frac{\pi^{2}}{3}\left(\frac{k_{\mathrm{B}}}{\mathrm{e}}\right)^{2}$ denoting the Lorenz number for ideal metals. The difference between $\kappa$ and $\kappa_{\mathrm{el}}$ may be attributed to the heat carried by phonons accounting for a rather temperature-independent contribution of $\kappa_{\mathrm{ph}} \approx 2.1 \mathrm{~W} /(\mathrm{m} \mathrm{K})$. Similar values of $1.6 \mathrm{~W} /(\mathrm{m} \mathrm{K})$ in semiconducting, needle-like crystals of functionalized pentacenes have been determined along the stacking direction ${ }^{[27]}$. The large 
apparent $\kappa_{\mathrm{ph}}$ may also point to an increased $L$ as predicted and experimentally verified for quasi-onedimensional Luttinger liquids ${ }^{[10,28]}$. In $\mathrm{DCNQI}_{2} \mathrm{Cu}$ however, a weaker temperature-dependence of $\kappa$ than expected from the WF law is observed. In addition, the calculated electronic contribution exceeds the experimental data upon approaching the Debye temperature of $\theta_{\mathrm{D}} \approx 82 \mathrm{~K}^{[26]}$ defining the lower validity limit of the WF law due to different relaxation times for thermal and electric field perturbations of the electronic system. Considering an additional phonon contribution on the order of $1 \mathrm{~W} /(\mathrm{m} \mathrm{K})$ the WF law seems to be violated even far above $\theta_{\mathrm{D}}$. Hence, other effects emerging from the low dimensionality of the materials seem to come into play, the details of which are currently investigated. The lattice thermal conductivity should reveal a temperature dependence similar to the phonon drag part of the thermopower since both processes are limited by phonon-phonon Umklapp scattering above a small fraction of the Debye temperature. At first glance, the drop in thermal conductivity below $40 \mathrm{~K}$ seems contradictory to the peak in the thermopower. Yet, the data is superimposed by the electronic contribution to heat conduction and the decrease is only evidenced by one data point. Hence, the drop in $\kappa$ at this temperature is disregarded in the subsequent conservative estimation of the thermoelectric performance. Boundary scattering may be neglected in the investigated temperature regime according to the calculated scattering rate of $\tau_{b d}^{-1} \leq 10^{8} s^{-1}$ being small compared to the phonon relaxation rate of $\tau_{b d}^{-1} \approx 10^{11} s^{-1}$ estimated from the thermal conductivity at $40 \mathrm{~K}$ (compare supplementaries). A detailed discrimination of lattice and electronic contributions to the thermal conductivity will be provided in a forthcoming paper.

At RT, the measured electronic properties result in power factors of $387 \mu \mathrm{W} /\left(\mathrm{m} \mathrm{K}^{2}\right)$ and $110 \mu \mathrm{W} /\left(\mathrm{m} \mathrm{K}^{2}\right)$ for $\mathrm{TTT}_{2} \mathrm{I}_{3}$ and $\mathrm{DCNQI}_{2} \mathrm{Cu}$, respectively. These are similar to those of polymerbased thermoelectrics ${ }^{[4]}$ but were obtained without additional crystal doping. Even with the rather high thermal conductivity of organic single crystals we obtain figures of merit reaching $z T_{\mathrm{TTT}_{2} \mathrm{I}_{3}}=$ 0.03 and $z T_{\mathrm{DCNQI}_{2} \mathrm{Cu}}=0.02$ which to our knowledge are the highest values consistently determined on single organic crystals so far ${ }^{[3]}$. Taking further control over the charge transfer process, e.g. by intentionally alloying of $\mathrm{DCNQI}_{2} \mathrm{Cu}_{x} \mathrm{Li}_{1-\mathrm{x}}{ }^{[29]}$ or reducing the iodine concentration in $\mathrm{TTT}_{2} \mathrm{I}_{3}{ }^{[25]}$, might 
enable a distinct manipulation of the charge carrier density. This should allow, in principle, for $z T$ values comparable to or even beyond those of polymers and inorganic thermoelectrics. The potential of the material class under study becomes obvious when looking at the temperature dependence of the power factors as well as at the individual and combined figures of merit in Figure 3d-e. Above $100 \mathrm{~K}$, the $z T$ temperature-dependence of both materials appears flat, enabling a broad temperature usability for single-stage devices. For $\mathrm{DCNQI}_{2} \mathrm{Cu}$ at low temperatures, the high power factor emerging from the phonon drag effect in the thermopower together with the anomalous strong temperature dependence of the resistivity render a $z T_{\mathrm{DCNQI}_{2} \mathrm{Cu}}(<40 \mathrm{~K}) \geq 0.15$ possible outperforming the, to our knowledge, maximum value of $z T=0.12$ obtained for $\mathrm{YbAgCu}_{4}$ in this temperature regime ${ }^{[30]}$. Therefore, thermoelectric materials based on small molecule radical ion salts can be expected to open up new fields of application, in low temperature thermoelectrics.

As a proof-of-concept, we finally built an organic single-junction thermoelectric generator with $\mathrm{TTT}_{2} \mathrm{I}_{3}$ as p-type and $\mathrm{DCNQI}_{2} \mathrm{Cu}$ as n-type conducting materials as illustrated in Figure 4a. The RT output characteristics of the generator are depicted in Figure $4 \mathrm{~b}$ for various temperature differences applied. The linear slope relating the open-circuit voltage to the applied temperature difference corresponds to the sum of the Seebeck coefficients obtained for $\mathrm{DCNQI}_{2} \mathrm{Cu}$ and $\mathrm{TTT}_{2} \mathrm{I}_{3}$, indicating an good retention of the temperature gradient across the crystals (see inset). Using only a single junction we reached power outputs of up to $125 \mathrm{nW}$ at $\Delta T=92 \mathrm{~K}$. The efficiency (supplementary information) reached values of $\eta_{\max }=0.017 \%$ and an effective $z T_{\text {eff }}=0.005$, being of the same order as the combined $z T_{\text {comb }}=0.026$ calculated from the individual crystals in Figure $3 \mathrm{e}$. We optimized the overall contact resistance down to values as small as $R_{\text {cont }} \approx 23 \Omega$ which still reduces the power output and $z T_{\text {eff }}$ of the generator. Going beyond the scope of this study, contact engineering will be essential for efficient organic thermoelectric devices manufactured in future. While the absolute efficiency values still require further improvement, it is worth looking at the specific power output normalized to the active cross-sectional area of both crystals, as depicted on the right ordinate of Figure $4 b$. This key parameter for cost-per-watt in waste heat recovery ${ }^{[31]}$ reaches values of 
$1 \mathrm{~mW} / \mathrm{cm}^{2}$ at $\Delta T=30 \mathrm{~K}$ being three orders of magnitude larger than those of thermoelectric devices based on polymers ${ }^{[2]}$.

To sum up, organic thermoelectrics composed of crystalline low-dimensional molecular metals present a promising alternative to existing material concepts, especially considering ongoing research to take full control over the amount of charge-transfer and band filling ${ }^{[32]}$. The peculiar phenomena observed in this material class such as violation of the WF law, the anomalous temperaturedependence of the electrical conductivity or phonon drag effects in the thermopower, will furthermore unlock new possibilities in low-temperature thermoelectrics.

\section{Supporting Information}

Supporting Information is available from the Wiley Online Library or from the author.

\section{Acknowledgements}

F.H. and A.S. contributed equally to this work. Financial support by the Deutsche Forschungsgemeinschaft (Project No. PF385/6-1) and the Seventh Framework Programme (H2ESOT, Project No. 308768) of the European Commission are gratefully acknowledged. We would like to thank Anatolie Casian (Technical University of Moldova) and Markus Schwoerer (University of Bayreuth) for fruitful discussions and helpful comments.

Received: ((will be filled in by the editorial staff))

Revised: ((will be filled in by the editorial staff)) Published online: ((will be filled in by the editorial staff))

\section{References}

[1] C. Forman, I. K. Muritala, R. Pardemann, B. Meyer, Renew. Sustain. Energy Rev. 2016, 57, 1568.

[2] Y. Sun, P. Sheng, C. Di, F. Jiao, W. Xu, D. Qiu, D. Zhu, Adv. Mater. 2012, 24, 932.

[3] J. Wüsten, K. Potje-Kamloth, J. Phys. D. Appl. Phys. 2008, 41, 135113.

[4] O. Bubnova, Z. U. Khan, A. Malti, S. Braun, M. Fahlman, M. Berggren, X. Crispin, Nat. Mater. 2011, 10, 429.

[5] O. Bubnova, Z. U. Khan, H. Wang, S. Braun, D. R. Evans, M. Fabretto, P. Hojati-Talemi, D. Dagnelund, J.-B. Arlin, Y. H. Geerts, S. Desbief, D. W. Breiby, J. W. Andreasen, R. Lazzaroni, W. M. Chen, I. Zozoulenko, M. Fahlman, P. J. Murphy, M. Berggren, X. Crispin, 
Nat. Mater. 2014, 13, 190.

[6] G. J. Snyder, E. S. Toberer, Nat. Mater. 2008, 7, 105.

[7] B. Poudel, Q. Hao, Y. Ma, Y. Lan, A. Minnich, B. Yu, X. Yan, D. Wang, A. Muto, D. Vashaee, X. Chen, J. Liu, M. S. Dresselhaus, G. Chen, Z. Ren, Science 2008, 320, 634.

[8] R. Venkatasubramanian, E. Siivola, T. Colpitts, B. O’Quinn, Nature 2001, 413, 597.

[9] L. D. Hicks, M. S. Dresselhaus, Phys. Rev. B 1993, 47, 12727.

[10] A. Garg, D. Rasch, E. Shimshoni, A. Rosch, Phys. Rev. Lett. 2009, 103, 96402.

[11] N. Stojanovic, D. H. S. Maithripala, J. M. Berg, M. Holtz, Phys. Rev. B 2010, 82, 75418.

[12] J. Vavro, M. C. Llaguno, J. E. Fischer, S. Ramesh, R. K. Saini, L. M. Ericson, V. A. Davis, R. H. Hauge, M. Pasquali, R. E. Smalley, Phys. Rev. Lett. 2003, 90, 65503.

[13] S. M. Kauzlarich, S. R. Brown, G. J. Snyder, Dalton Trans. 2007, 2099.

[14] X. Shi, J. Yang, L. Wu, J. R. Salvador, C. Zhang, W. L. Villaire, D. Haddad, J. Yang, Y. Zhu, Q. Li, Sci. Rep. 2015, 5, 14641.

[15] A. Casian, Phys. Rev. B 2010, 81, 155415.

[16] T. Mori, A. Kobayashi, Y. Sasaki, H. Kobayashi, Bull. Chem. Soc. Jpn. 1983, 56, 3376.

[17] A. Casian, V. Dusciac, I. Coropceanu, Phys. Rev. B 2002, 66, 165404.

[18] R. Kato, S. Aonuma, H. Sawa, Synth. Met. 1995, 70, 1071.

[19] P. M. Chaikin, J. F. Kwak, Rev. Sci. Instrum. 1975, 46, 218.

[20] C. Dames, G. Chen, Rev. Sci. Instrum. 2005, 76, 1.

[21] L. Lu, W. Yi, D. L. Zhang, Rev. Sci. Instrum. 2001, 72, 2996.

[22] A. Matsui, Y. Takaoka, Y. Nishio, R. Kato, K. Kajita, J. Phys. Conf. Ser. 2009, 150, 42120.

[23] K. Torizuka, H. Tajima, T. Yamamoto, J. Phys. Soc. Japan 2006.

[24] T. Mori, H. Inokuchi, A. Kobayashi, R. Kato, H. Kobayashi, Phys. Rev. B 1988, 38, 5913.

[25] P. M. Chaikin, G. Grüner, I. F. Shchegolev, E. B. Yagubskii, Solid State Commun. 1979, 32, 1211.

[26] Y. Nishio, M. Tamura, K. Kajita, S. Aonuma, H. Sawa, R. Kato, H. Kobayashi, J. Phys. Soc. 
Japan 2000, 69, 1414.

[27] H. Zhang, Y. Yao, M. M. Payne, J. E. Anthony, J. W. Brill, Appl. Phys. Lett. 2014, 105, 73302.

[28] N. Wakeham, A. F. Bangura, X. Xu, J.-F. Mercure, M. Greenblatt, N. E. Hussey, Nat. Commun. 2011, 2, 396.

[29] K. Hiraki, K. Kanoda, Synth. Met. 1997, 86, 2111.

[30] M. Koirala, H. Wang, M. Pokharel, Y. Lan, C. Guo, C. Opeil, Z. Ren, Nano Lett. 2014, 14, 5016.

[31] D. M. Rowe, G. Min, J. Power Sources 1998, 73, 193.

[32] Y. Krupskaya, I. G. Lezama, A. F. Morpurgo, Adv. Funct. Mater. 2016, 26, 2334.
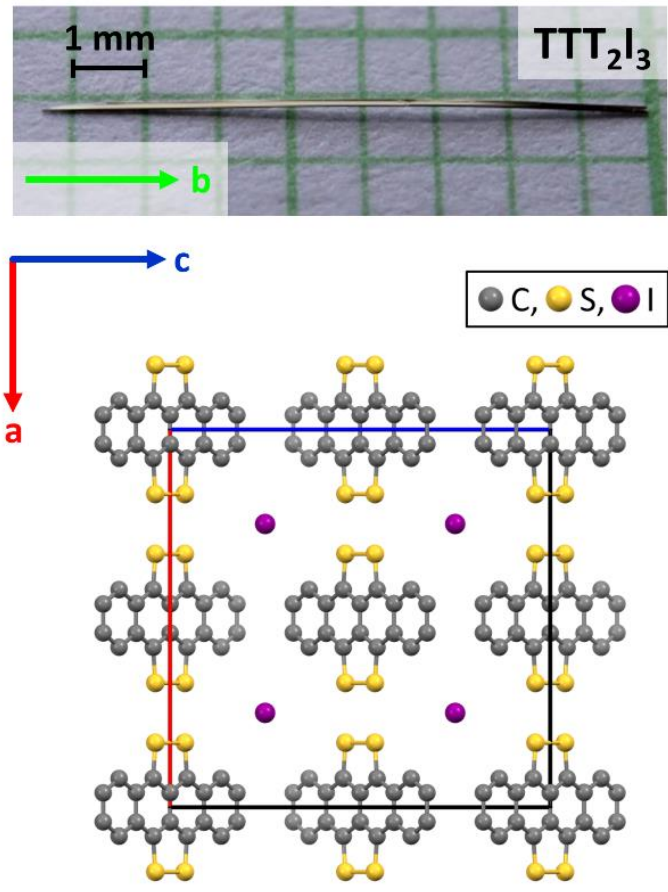

b
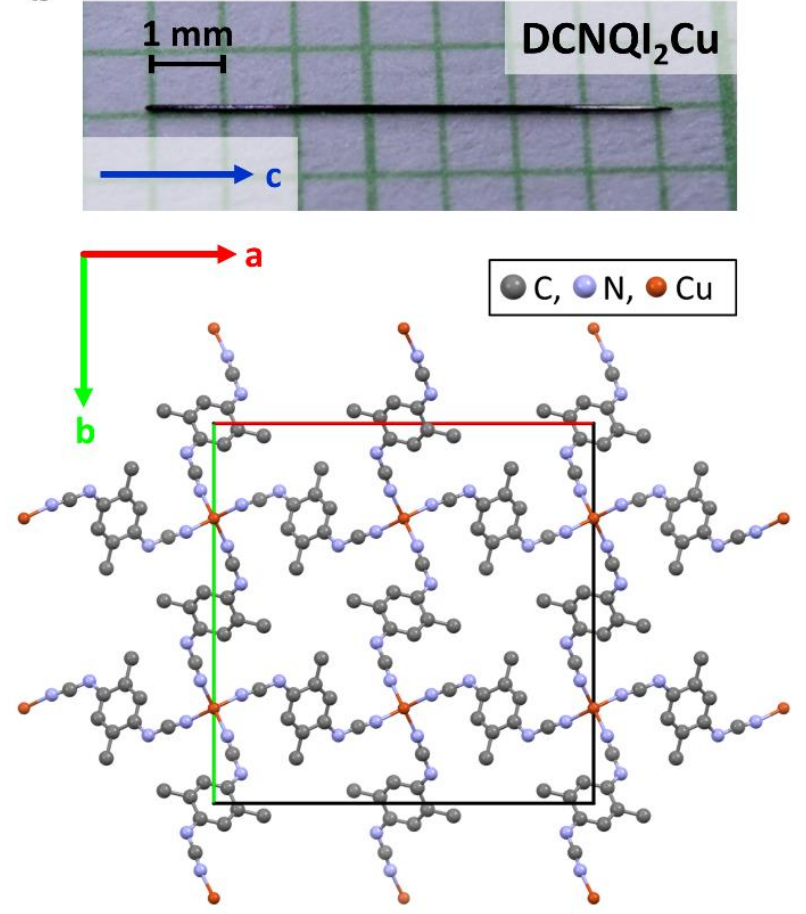

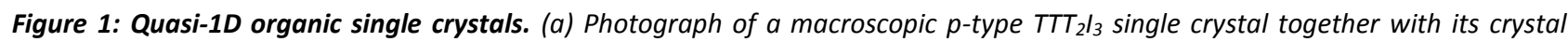
structure showing the separated molecular stacks. (b) The same is illustrated for the investigated crystalline n-type organic conductor ${ }_{D C N Q I} \mathrm{Cu}$. 
a

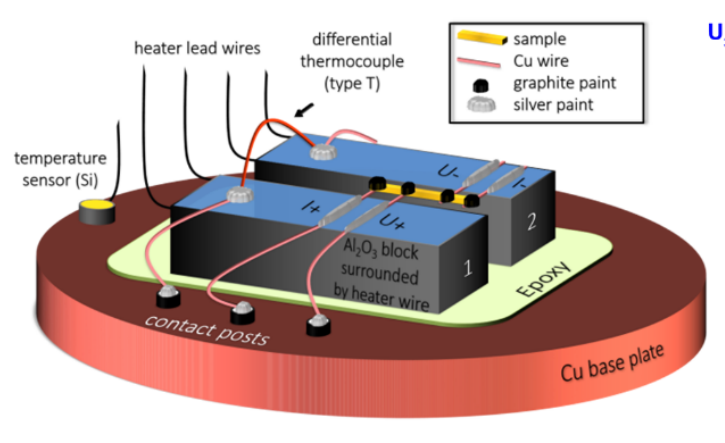

b

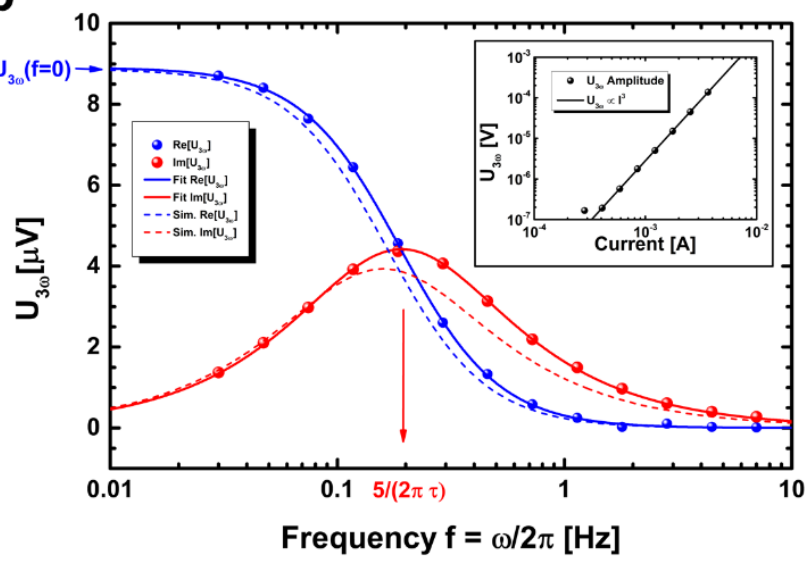

C
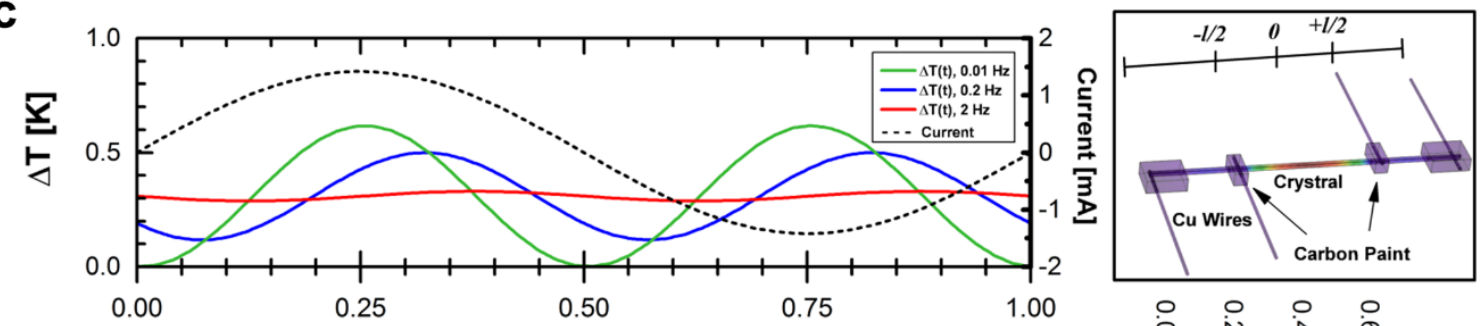

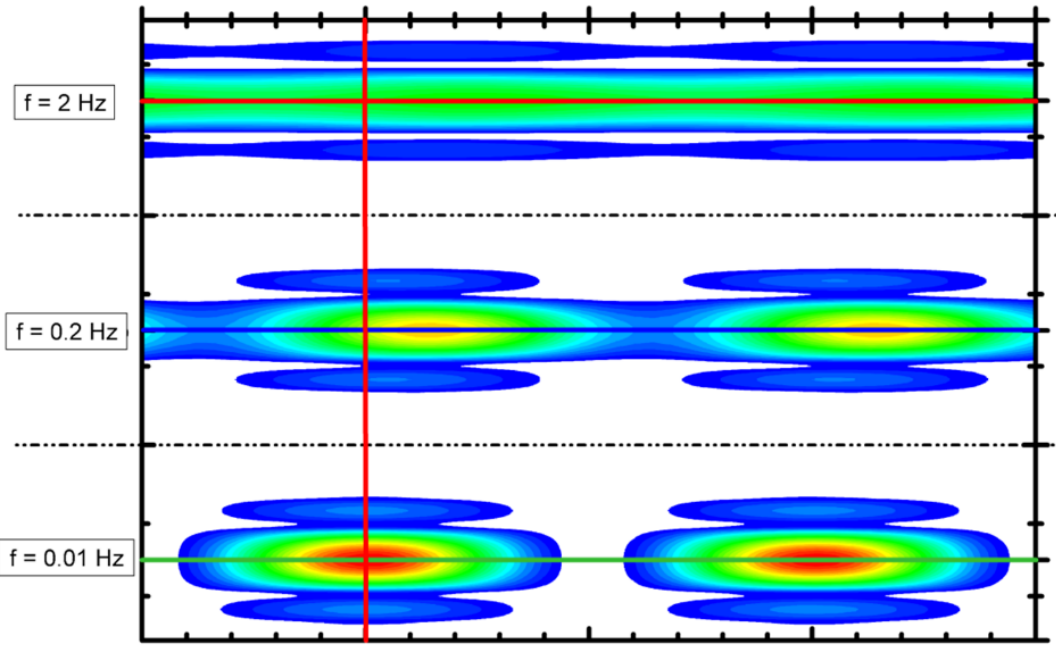

Normalized Time $[\mathrm{t} / \mathrm{T}]$

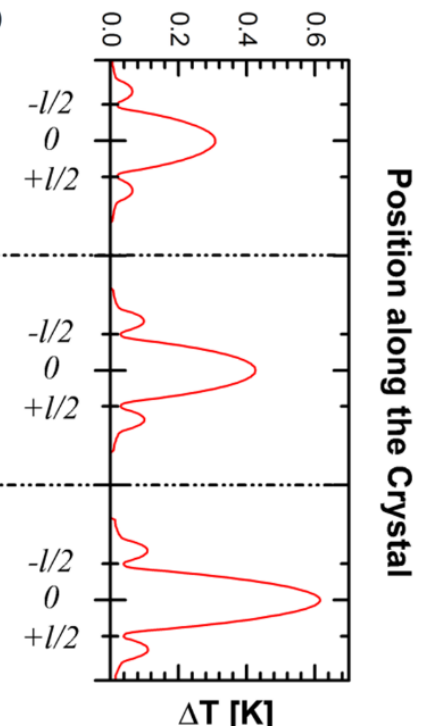

$\Delta \mathbf{T}[\mathrm{K}]$

Figure 2: Self-Heating 3w-technique. (a) Sample holder utilized to measure all three thermoelectric quantities on the same crystal attached to four free-standing copper leads, thermally connected to two independent heating blocks. (b) Measured and simulated real and imaginary part of the $3 \omega$-voltage. The thermal conductivity is determined from the amplitude of the real part at zero frequency while the peak position in the imaginary part corresponds to $5 /(2 \pi \tau), \tau=4.0 \mathrm{~s}$ being the thermal relaxation time of the crystal. The cubic current dependence of the total $3 \omega$-voltage, as anticipated by Equation 2, is depicted in the inset for $f=0.1 \mathrm{~Hz}$. Especially at low frequencies, the simulated $3 \omega$-voltage agrees well with the measurement enabling an accurate determination of $\kappa$. This was derived from the simulated temporal evolution of the temperature profile along the crystal, as depicted for three different frequencies in (c). The thermal conductivity and specific heat as determined by fitting the experimental data have been used as material properties. The temperature profiles between the contacts at $\pm l / 2$ are quasi-parabolic. While the temperature oscillation follows the current amplitude for small frequencies, a phase-shift occurs for $f \approx 5 /(2 \pi \tau)$. Moreover, the temperature rise does not relax back to zero at vanishing current amplitude anymore. At high frequencies the oscillation amplitude is decreasing, corresponding to DC heating of the crystal to a constant temperature offset by the effective power $U_{R M S} \cdot I_{R M S}$. 

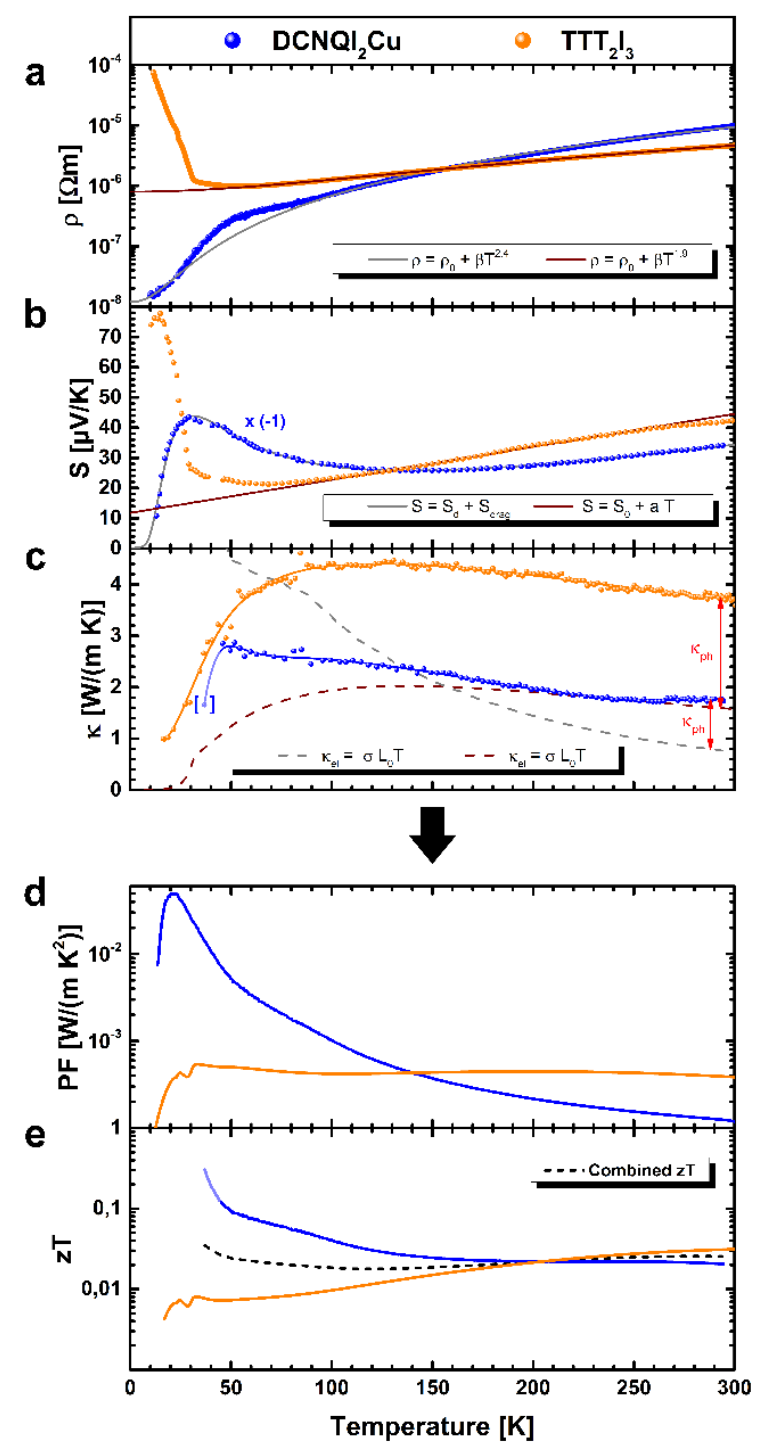

Figure 3: Thermoelectric properties of $\mathrm{TTT}_{2} \mathrm{I}_{3}$ (p-type) and $\mathrm{DCNQI}{ }_{2} \mathrm{Cu}$ (n-type) crystals. (a) The temperature dependence of the electrical resistivity $\rho=\rho_{0}+\beta T^{\gamma}$, with $\gamma>1$, for both materials is typical for low-dimensional organic metals. (b) Absolute values of the Seebeck coefficient $S$. The linear fit in the metallic regime of $T_{T T} I_{3}$ yields a band width of $632 \mathrm{meV}$. In the case of DCNQI ${ }_{2} \mathrm{Cu}$, an additional contribution of the phonon drag is considered (Equation 3) resulting in $W_{D C N Q I_{2} C u}=333$ meV. (c) Temperature dependence of the thermal conductivity $\kappa$, determined by means of $3 \omega$-measurements. In addition, the electronic thermal conductivity $\kappa_{e l}$ as calculated by the WF law is shown. The phonon contributions $\kappa_{p h}$ at $R T$ for $\mathrm{TTT}_{2} \mathrm{I}_{3}$ and $\mathrm{DCNQI} \mathrm{I}_{2} \mathrm{Cu}$ are roughly estimated to $2.1 \mathrm{~W} /(\mathrm{m} \mathrm{K})$ and $1 \mathrm{~W} /(\mathrm{m} \mathrm{K})$, respectively. The quantities $\rho, S, \kappa$, all of which were measured on the same respective specimen, result in (d) the power factor $S^{2} / \rho$ and (e) the dimensionless figure of merit $z T$. 
a

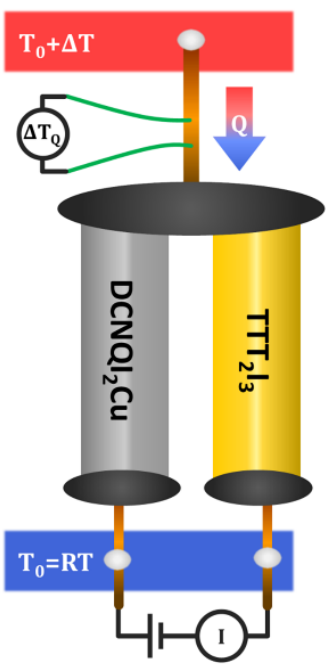

b

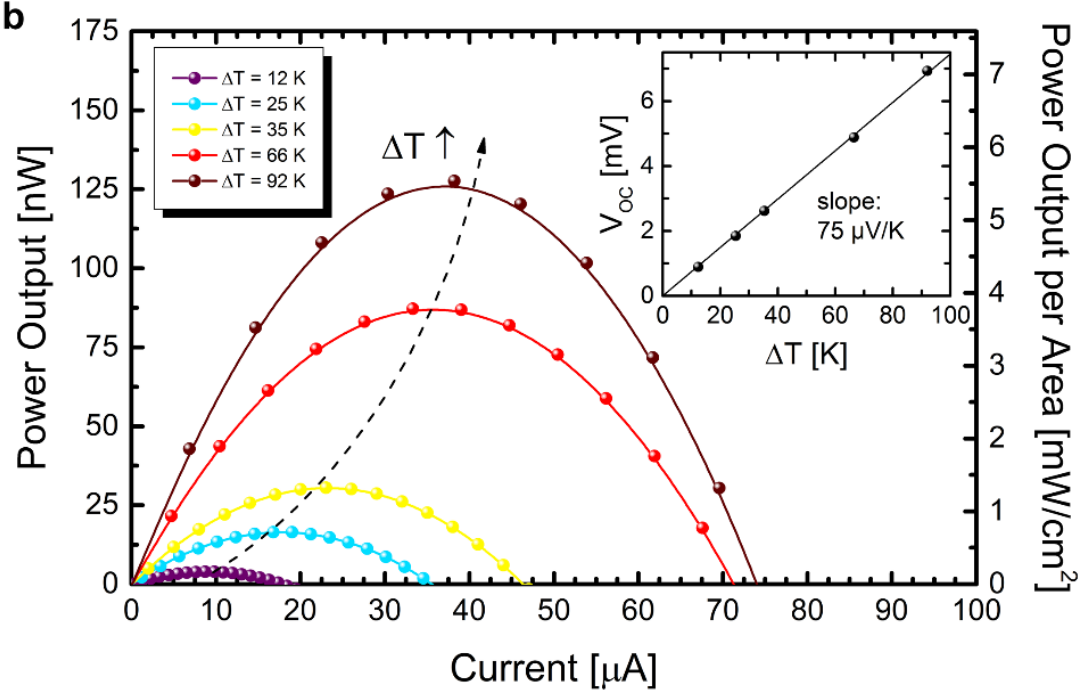

Figure 4: Organic thermoelectric generator. (a) Scheme of a prototypical device build-up of n-type DCNQI ${ }_{2} \mathrm{Cu}_{\text {and }}$ p-type $T T T_{2} I_{3}$ legs. Crystals are electrically contacted with graphite paint. The common contact of the two crystals was heated via a copper wire attached to a heating block. The opposite electrical contacts were attached to an alumina block at RT and connected to the measurement circuit. At various temperature differences the current-voltage curves were recorded resulting in (b) the power output characteristics. Normalized to the overall cross section of both crystals the resulting high power output per area reveals values demonstrating the potential of organic thermoelectrics. The inset reveals the open circuit voltage to scale linearly with the temperature difference applied. 